\title{
Das Mystische im Konzept der Methode des Philosophierens bei Rosenzweig und Heidegger
}

\section{Martin Brasser}

\section{(2) OpenEdition}

1 Journals

Édition électronique

URL : https://journals.openedition.org/cps/2686

DOI : $10.4000 /$ cps.2686

ISSN : 2648-6334

Éditeur

Presses universitaires de Strasbourg

\section{Édition imprimée}

Date de publication : 1 juin 2011

Pagination : 203-212

ISBN : 978-2-354100-36-0

ISSN : $1254-5740$

Référence électronique

Martin Brasser, „Das Mystische im Konzept der Methode des Philosophierens bei Rosenzweig und Heidegger", Les Cahiers philosophiques de Strasbourg [Online], 29 | 2011, Online erschienen am: 15 Mai 2019, abgerufen am 09 November 2022. URL: http://journals.openedition.org/cps/2686 ; DOI: https:// doi.org/10.4000/cps.2686

\section{c) (i) (3)}

Creative Commons - Namensnennung - Nicht-kommerziell - Weitergabe unter gleichen Bedingungen 4.0 International - CC BY-NC-SA 4.0

https://creativecommons.org/licenses/by-nc-sa/4.0/ 


\section{Das Mystische im Konzept der Methode des Philosophierens bei Rosenzweig und Heidegger}

Martin Brasser

Rosenzweigs Denken ist schon oft mit demjenigen Heideggers verglichen worden. Den Anfang dazu hatte Rosenzweig mit seiner Stellungnahme zur Davoser Disputation selbst gemacht. Auf den ersten Blick fällt auf, dass beide Philosophien auf etwas ausgerichtet sind, was jenseits der Philosophie liegt und im Prinzip auch ohne die Philosophie erlangt werden kann. Dazu suchen beide für die Philosophie nach der Methode, die zu diesem für die Philosophie wesentlichen Charakter des Ausgreifens über sich selbst hinaus am besten passt.

\section{I. Über altes, falsches, neues und richtiges Denken}

Wenn Mystik die Suche ist nach dem, was immer jenseits liegt, dann sind Rosenzweig und Heidegger "Mystiker des Denkens». Heidegger wollte eine Mystik des Denkens haben, die ganz ohne Theologie auskommt und nur durch das Denken selbst erschlossen wird. Dafür sollte man nicht nur die Vorstellung ewiger Wahrheiten, sondern die Art von Denken insgesamt hinter sich lassen, die aus Sicht Heideggers «zu den längst noch nicht radikal ausgetriebenen Resten von christlicher Theologie innerhalb der philosophischen Problematik» (Heidegger 1986, S. 229) gehört. Heidegger bezeichnet diesen Rest eine Zeit lang als «Metaphysik», später als «Onto-theologie» (Heidegger 1990). Ihren Wesenszug meint er im Vorstellen ausfindig zu machen, das - wieder aus seiner Sicht - schon in dem Moment in die Denkgeschichte eingedrungen war, als zum ersten Mal das, was eine Idee ist, so gedacht wurde, wie man die Gleichung « $A=B$ » dann 
denkt, wenn man beide Glieder dieser Gleichung bereits als bekannt voraussetzen darf. " $A=B$ » besagt, dass sich das, was wir uns unter " $A$ " vorstellen, so nach dem richtet, was wir uns auch unter « $\mathrm{B}$ » vorstellen und umgekehrt, so dass wir deshalb sagen können: beide sind gleich. «A» richtet sich dann gewissermaßen ganz nach « $B$ » und umgekehrt. Das Konzept einer so verstandenen Idee enthält folglich das Prinzip der Ausgerichtetheit-nach-einem-bestimmten-Vorgegebenem - und dadurch wird, so Heidegger, bereits eine ganz bestimmte Ordnung etabliert: das Konzept der Idee wird dem Konzept von Richtigkeit unterworfen (Heidegger 1976, S. 230f). Entsprechend muss dann im Gegenzug das nicht-metaphysische Denken genau diese Unterwerfung aufgeben und seine neuen Paradigmen aus solchen Bereichen nehmen, die sich nicht der Orientierung an die Idee der bestimmten Ausrichtung, also der Vorstellung und Richtigkeit verdanken. Um nicht in diese Falle zu tappen, muss Philosophie deshalb neben all ihren anderen Aufgaben auch die Frage nach der Art, wie das spezifisch philosophische Denken anders denn vorstellend vor sich geht, beantworten können. Weil die Falle fortbesteht, ist die Einleitung in die Philosophie ein immer wieder neu vorzunehmendes Unternehmen der Philosophie selbst. Darin wird jeweils die Frage nach der Methode gestellt, nach der das Denken organisiert ist, wenn man es mit Recht «Philosophie» nennen können will.

Auch Franz Rosenzweig schickt die Philosophie auf den Weg zu einer neuen, historisch noch nicht entwickelten Methode, derer sie bedarf, wenn sie den mystischen Schritt über das Denken hinaus vollziehen will. Wie bei Heidegger liegt dieses "gelobte Land» der Philosophie jenseits der Philosophie selbst. Um zu einem Denken zu gelangen, das sich über diese Positionierung der Philosophie als «mystischem Denken" im Klaren ist, bedarf es auch nach Rosenzweig der Überwindung der Tendenz der Philosophie, sich den Weg aus sich selbst heraus zu verbauen. Die Tendenz zur Verhinderung der Überschreitung auf «jenseits» ist das Wesensmerkmal des von Rosenzweig so genannten "alten Denkens», während das neue Denken die Orientierung auf das, was über das Denken hinausgeht, aufrecht erhält oder überhaupt erst eröffnet. Die Diagnose des Irrwegs ist für Rosenzweig rasch gemacht: wo immer Philosophie auftrat, trat sie mit dem Anspruch auf, systematische Erkenntnis von allem und des Gesamten zu sein. Diese Tendenz zum System ist für sich schon der Irrweg, denn Systeme tendieren ihrer 
inneren Dynamik nach darauf, allem, was es gibt, einen Platz im Ganzen zuzuweisen. Damit hat sich ein solches Denken bereits anders ausgerichtet, als es von dem oben so bezeichneten «mystischen Denken" zu erwarten wäre, nämlich als Bezug auf all das, dem prinzipiell immer ein Platz innerhalb des Denkens zugewiesen werden kann. Dass man unter dieser Bedingung auf das Denken gleich ganz verzichten zu müssen meint, wenn man den Bereich erreichen will, der in dieser Terminologie "das Mystische» genannt werden kann, ist selbst nur Ausdruck des alten Denkens, innerhalb dessen man sich eine Alternative zum systembildenden Vorgehen nur dann vorstellen kann, wenn diese Alternative selbst nicht als Denken auftritt. Deshalb muss das neue Denken methodisch so organisiert sein, dass es einerseits nicht in die Systematisierung hinein- und andererseits auch nicht aus dem Denken selbst herausfällt.

Beide Male, bei Heidegger und bei Rosenzweig, ist die Philosophie im Grunde ein Wegbereiter, eine Durchgangsstation oder ein Türöffner in einen Raum, der selbst nicht mit Kategorien erfasst wird, die typisch sind für unser Verständnis von Denken als aktivem Sich-auf-sichselbst-Beziehen (reditio completa ad se ipsum). Für die Beschreibung der Methode, wie man auf diesen anderen Raum denkend zugreift, passen Kategorien aus der Tugendlehre wie Demut und Bescheidenheit oder aus der einfachen Wahrnehmungslehre wie Hinhören und Stille oder aus der Praxis gelungener Lebensführung wie Gelassenheit und «(einfachhin) leben" offensichtlich viel besser. Beide Male gehen diese letzteren Kategorien aber nicht auf etwas, das sich abspielt, sobald das Denken an seinen Anschlag gekommen ist. Sondern sowohl Rosenzweig als auch Heidegger gehen davon aus, dass das Denken an diesen Kategorien entlang entfaltet wird, gerade wenn es ganz bei sich ist und zu sich selbst gekommen ist.

Es gibt also in der Philosophie zwei prinzipiell unterschiedliche Philosophien oder zwei Verständnisse von Denken. Das eine ist das eigentliche, richtige und gesollte - und das andere ist das falsche. Es gibt folglich zwei Begriffe von Philosophie und damit zwei Wege, wie man Philosophie betreiben kann. Die Methode dieses Betreibens macht den Unterschied zwischen dem "richtigen» und dem "falschen » Denken.

Es gehört zu den großen Gesten großer Philosophie (ist aber kein Kriterium für ihre Unterscheidung gegenüber «kleiner» Philosophie), dass sie sich ihrem Publikum nicht nur als das neue, sondern auch als 
das richtige zu vermitteln versucht, indem es das bisherige als das falsche und irrläufige diagnostiziert. Diese Geste ist so alt wie die Philosophie selbst. Für Heidegger und für Rosenzweig ist darüber hinaus aber anders als für viele "Verfallstheoretiker» vor ihnen auch klar: diese Tendenz zur Verbiegung der Philosophie ist eine im Denken selbst angelegte und deshalb unvermeidbare, aber durch das Denken selbst im Ansatz durchschaubare Tendenz. Wesentliche Teile ihrer eigenen Philosophie widmen sie dem Versuch, diese Tendenz zu durchschauen. Sie setzen dabei beide an derselben Stelle ein: bei der Arbeit an den Grundbegriffen.

\section{Die Arbeit an den Grundbegriffen}

Philosophie ist mehr als bloß ein Instrument, mit dem man sicherstellt, dass Aussagen universal nachvollziehbar sind. Sie ist Arbeit an ihrer eigenen Justierung und als solche bereits Philosophie. Diese Justierung bezieht sich, wie gesehen, auf die eigene Herkunft, gegen die sich abzusetzen die Aufgabe ist. Sie bezieht sich aber natürlich auch auf den Gegenstand, mit dem sie sich beschäftigen will. Auch hier haben Heidegger und Rosenzweig vieles gemeinsam. Beide arbeiten an den Grundbegriffen. Diese sollen im Sinn der Rejustierung des Denkens ihrerseits neu gefasst werden, damit das Ziel der Philosophie erreicht werden kann, sich selbst auf das zu überschreiten, was einmal - nach Heidegger - als die "Wahrheit des Seins" affirmiert oder - bei Rosenzweig - als «Leben" gelebt wird. Diese Arbeit an den Grundbegriffen hat immer korrigierende Auswirkungen auf alles, was auf diesen Grundbegriffen aufbaut. Das ist zunächst die Philosophie selbst, aber auch alle anderen Wissenschaften, mit deren jeweiligem Grundbegriff man sich im Rahmen der Revision der «alten » Philosophie beschäftigt. Die Arbeit an den Grundbegriffen hat folglich immer auch wissenschaftskonzeptionelle Auswirkungen. Konkret hat Heidegger dies für das Verständnis von Theologie exemplarisch durchzuführen versucht. Er trifft sich hier mit Rosenzweigs Überzeugung, dass dessen eigenes neues Denken insbesondere die Theologie, und hier natürlich wieder insbesondere die jüdische Theologie verändern wird.

Wirkungsgeschichtlich ist das sicher der Fall geworden. Von Rosenzweig selbst war es darüber hinaus aber auch methodischkonzeptionell gemeint, wenn er an den Grundbegriffen arbeitete, die 
der jüdischen Theologie zugrunde liegen: Gott, Welt und Mensch. In Rosenzweigs eigenen Worten heißt dies so: "das Jüdische ist meine Methode, nicht mein Gegenstand» (Rosenzweig 1979, S. 720). Was das Methodische am Jüdischen ist, ist damit zunächst nicht gesagt. Es wird aber klarer, wenn man sich das Prozedere vor Augen führt, mit dem Rosenzweig die Grundbegriffe bearbeitet, auf denen die Philosophie, die Wissenschaft und das Denken überhaupt aufbauen. Mit dem Terminus "De(kon)struktion" ist diese Methode formal korrekt beschrieben. In der Sache geht es um eine bestimmte Art, die Idee des Ereignisses in die Konzeption der Grundbegriffe zu integrieren.

Wie bei Heidegger geht es tendenziell auch für Rosenzweig darum, den sachlichen Gehalt eines jeweiligen Grundbegriffs zunächst bis auf den Kern freizuschälen (Destruktion) und dann auf der Kernbedeutung das neue Denken aufzubauen (Konstruktion). Beide wissen auch, dass die Intention selbst in der Tendenz unendlich ist, man also beim Weg des "Schälens» schwerlich je an einen Punkt kommt, den man legitimerweise als den Kern bezeichnen darf. Rosenzweig hat hierfür das Bild von den Grundbegriffen als den "Zwiebeln, die man schälen kann, soviel man will, - man kommt immer nur auf Zwiebelblätter und nicht auf etwas ,ganz anderes'" (Das neue Denken, in Rosenzweig 2001, S. 215), sprich einen Kern. Diese Endlosigkeit sollte man streng methodisch nehmen. Sie bedeutet, dass der Ansatz für den Aufbau selbst nicht aus dem Ergebnis der Destruktion herrühren kann. Denn selbst wenn man faktische «Kernbestände» von Grundbegriffen herausarbeiten könnte, wäre ein solches Ergebnis methodisch gesehen nur ein Vor-Ergebnis und käme als Ausgangspunkt für die Konstruktion nicht in Frage. Der Angelpunkt des Ausgangspunkts liegt jenseits der Grundbegriffe. Das wissen sowohl Heidegger als auch Rosenzweig. Dieser Angelpunkt ist vor der Destruktion bereits da. Rosenzweig hat hierfür das Bild vom Kuchenbacken: "In ein Erkennen, bei dem etwas herauskommt, ist genau wie bei einem Kuchen auch etwas hineingetan" (Das neue Denken, in Rosenzweig 2001, S. 231).

In der Art, wie sich der Aufbau vollzieht, liegt das methodische Novum des neuen Denkens. Schon beim ersten methodischen Schritt, der Destruktion, wird klar, wie sehr jeder der Grundbegriffe, die Rosenzweig analysiert (Gott, Welt und Mensch), einen Anteil in sich trägt, der nicht von irgend einem System je verortet oder gar in ein größeres Ganzes eingebunden werden kann. Das ist aus dem Konzept, das die Philosophie 
insgesamt in eine Erfolgs- und eine Verfallsgeschichte eingeteilt ist, kaum anders zu erwarten. Dieses Konzept wird hier auf der Ebene der Begriffe und ihrer Strukturen noch einmal wiederholt. Die Grundbegriffe sind folglich wie die Philosophie selbst notwendig zweigeteilt. Man kann das sogar noch genauer sagen: ein Teil davon ist systemfähig, der andere ist es nicht. Nur beide Teile zusammen machen den ganzen Grundbegriff aus. Systemorientiertes Denken blendet immer den einen der beiden Anteile an einem jeweiligen Grundbegriff $a b$ und bleibt deshalb entgegen aller eigenen Beteuerungen und dem eigenen Ansatz zum Trotz nicht nur okkasionell, sondern prinzipiell undurchführbar. Was dann die Kohärenz eines jeden Grundbegriffs in sich selbst und im Idealfall die Kohärenz aller Grundbegriffe untereinander herzustellen vermag, kommt von einer Stelle oder Struktur oder wie immer man es nennen will, die ihre systematisierende Kraft nicht daraus nimmt, dass sie alle Grundbegriffe ihrerseits übergreift.

Blickt man auf die destruktive Komponente an der Methode der Philosophie, sieht man ziemlich schnell, worin diese systematisierende Kraft in Tat und Wahrheit besteht: jeder Grundbegriff hat seine verschiedenen Bedeutungsschichten, weil er selbst eine Geschichte hinter sich hat. Jeder Grundbegriff ist zeitlich geworden. Der Abtrag der «Zwiebelschichten» ist ein Rückgang hinter den Prozess der Ablagerung der Bedeutungsschichten - und sei es nur der historisch eher grob angelegte hinter das "alte Denken" von "Jonien bis Jena» oder von Platon bis Nietzsche. Bei beiden Grobgesten gegen den mainstream der Tradition geht es um die Implementierung eines systematischen Neuansatzes. Zeit ist hier im Grunde nicht historisch gedacht - ihr Charakter als historische ist für Heidegger ohnehin erst ein abgeleitetes Phänomen. Sondern Zeit ist hier das Faktum des Auseinanderseins der drei Elemente der Zeit, der Vergangenheit, Gegenwart und Zukunft. Wie Heidegger zieht auch Rosenzweig aus dieser Idee von Zeit die Anleitung für die Antwort auf die Frage, wie die Philosophie methodisch durchgeführt werden muss, wenn sie erneuert werden will. Über die Methode des Sterns der Erlösung resümiert Rosenzweig: «So entspringen [sic M.B.] der Zeitlichkeit des neuen Denkens seine neue Methode» (Das neue Denken, in Rosenzweig 2001, S. 223). 


\section{Zeit als Struktur oder als Entzug}

Bei näherem Hinsehen gibt es freilich einen Unterschied zwischen der Art, wie Rosenzweig und wie Heidegger die Zeit methodisch auswerten. Dieser Unterschied erscheint je nach Perspektive groß oder klein. Das Auseinandersein der drei Elemente der Zeit fasst jeder der beiden unterschiedlich. Rosenzweig nähert sich dieser Ekstatik in der Zeit gewissermaßen rhetorisch: wenn man etwas erzählt, dominiert der Vergangenheits-, wenn man zu jemandem spricht, der Gegenwarts-, und wenn man gemeinsam (Psalmen) betet, der Zukunftsaspekt. Im zweiten, analytischen und von Rosenzweig gar nicht eigens herausgearbeiteten Schritt kann man dann klarmachen, dass diese unterschiedliche rhetorische Priorisierung eines Aspekts der Zeit gegenüber den jeweils beiden anderen nur deshalb möglich ist, weil die Zeit selbst «rein für sich» ein ekstatisches Auseinander ihrer drei Ekstasen darstellt. Diese Fundierungsebene ist bei Heidegger viel stärker herausgearbeitet als bei Rosenzweig, der umgekehrt viel stärker auf die Sprache und die Rede als methodischem Ausgangspunkt abhebt. Die flüssige systematische Verbindbarkeit beider Positionen täuscht aber nicht über die Unterschiede hinweg, die sich an dieser Stelle bis in die aktuelle Rezeptionstradition innerhalb der Rosenzweigforschung weiterführt und noch dort ausgetragen wird.

Aus einer «rosenzweigisierenden » Sicht gerät der Versuch, die Zeit als fundierendes Prinzip der Methode der Philosophie zu nutzen, in die Fänge der Systematisierung, aus der die Methode aber doch eigentlich hätte herausführen sollen. Die Zeit erhält das Aussehen einer Struktur, die sich auf allen Ebenen der Durchführung von Philosophie konstant durchhält und einen Grad von Wiedererkennung sicherstellt, der die methodische Einheitlichkeit des Neuen in der neuen Philosophie garantiert. So wird Zeit aber wie eine bleibende, feststehende Struktur behandelt - als wäre sie eine Zwiebel, die einen Kern hat. Das ist aus Sicht Rosenzweigs und Heideggers aber «altes Denken».

Aus einer «heideggerisierenden" Sicht ist jeder Versuch, das methodische Ideal der Verflüssigung aller grundbegrifflichen Gehalte konsequent anzuwenden und bis zur Endlosigkeit zu treiben, bei aller methodischen Gerechtfertigtheit ein denkerisches Kamikaze. Es hebt nicht nur die alte, sondern auch die neue Philosophie im Ansatz bereits 
auf. Auch das neue Denken muss systemaffin bleiben. Das ist aus Sicht Heideggers und auch aus derjenigen Rosenzweigs zwingend.

Hier streiten sich nicht zwei Positionen zweier Denker, sondern zwei Konsequenzen innerhalb derselben Option, die von beiden Denkern geteilt wird. Beide bejahen aufgrund ihres methodischen Konzepts von «Neuem Denken» beide Konsequenzen, ohne aber beide Konsequenzen gleichzeitig voll und ganz wollen zu können, weil die eine mit der anderen im Widerspruch liegt. Die Rezeptionsgeschichte hat es hier leichter. Man kann hier entweder auf die Werk- oder auf die Personalunion verzichten. Bei vielen Autoren, die sich in ihrem philosophischen Werk sowohl auf Rosenzweig als auch auf Heidegger beziehen, kann man beobachten, dass sie in ihrem eigenen Werk das eine Mal stärker auf die Idee einer übergreifenden Struktur (in der Idee der Zeit, der Andersheit und dgl.) und das andere Mal stärker bei der Uneinholbarkeit (in der Idee Zeit, der Andersheit und dgl.) ansetzen. Lévinas scheint mir hier das Beispiel par excellence zu sein. Wenn man von der Personalunion absieht, wird diese Weichenstellung in der Rosenzeigrezeption sogar noch augenfälliger. Hier kann der eine Rezipient die system-, der andere die prozessaffinere Position favorisieren, auch wenn sie denselben Ausgangspunkt beim Ereignischarakter der Zeit nehmen ${ }^{1}$.

Aber das täuscht freilich nicht darüber hinweg, dass die Methode des «Neuen Denkens» bei Rosenzweig oder mit Heidegger diejenige des nachmetaphysischen Denkens in sich selbst so verfasst ist, wie die Interpretation der Zeit es freilegt: als Widerstreit von Struktur und Entzug, als Gabe und Freiheit zugleich. Die Methode ist so verfasst wie die Zeit selbst, die so aussieht, als wäre sie eine Struktur, ohne dass sie es aber je sein kann, da sie ja "die Zeit» ist, die Strukturen erst ermöglicht. Was Rosenzweig über die Liebe sagt, kann man entsprechend auch auf die methodische Funktion der Zeit anwenden: sie erscheint so, als wäre sie die Struktur, in der sich das neue Denken gegen das alte methodisch neu verfasst - und das erscheint nur so, denn die Zeit selbst ist aller Fassung weit voraus. Aber «im Spiegel dieses Scheins spiegelt sich unmittelbar die Wahrheit» (Rosenzweig 1988, S. 224).

1 Ich denke hier konkret an die beiden zeitgleich publizierte Dissertationen: D. Fonti 2009 und M. del Prete 2009. 


\section{Ausblick auf das Jüdische an der Methode}

Damit ist im Prinzip die Antwort auf die Frage gegeben, worin das Jüdische an der Methode besteht, die Rosenzweig ähnlich wie Heidegger in die Philosophie neu einzuführen versucht hat. Das Jüdische wäre dann dieser Charakter des permanenten Wechsels oder sogar der prinzipiellen Ambivalenz zwischen Bestand und Entzug in dem, was man als Methode jeweils festhält und weitergibt. Eine Lektüre der Charakteristik des Judentums im «Stern der Erlösung» könnte, wie mir scheint, aufzuweisen versuchen, dass und wie diese Ambivalenz tatsächlich bestimmend ist für Rosenzweigs Konzeption des Judentums (mehr jedenfalls als für das Verständnis des Christentums). Eine solche Lektüre würde der hier vorgetragenen These vom Jüdischen als dem Methodisch-Ambivalenten in Rosenzweigs Versuch, in der Philosophie einen Neueinsatz zu stiften, sicherlich mehr Plausibilität verschaffen. Darüber hinaus liegt in diesem Konzept von Methode noch eine weitere Lektüreaufgabe. Die Nähe zu Heidegger - jedenfalls zu dem Heidegger, wie er Rosenzweig aus Davos übermittelt worden ist - hat seine zentrale Begründung darin, dass beide Philosophien die genannte Ambivalenz zur methodischen Grundlage erhoben haben. Die Zeit als gemeinsames Thema und die beide Philosophien verbindende Geste der Abstoßung von aller bisherigen Philosophie verdanken sich einem methodischen Ideal, das sowohl nach Heidegger als auch nach Rosenzweig in aller Philosophie bisher noch keine methodische Entfaltung gefunden hat. Es ist das Ideal der prinzipiellen Ambivalenz aller Erscheinung, das methodisch als Anspruch des Aufweises der inneren und unversöhnlichen Spannung in allen Grundbegriffen und zugleich als ein der gesamten bisherigen Philosophiegeschichte gegenüber neues Denken auftritt. Nur so scheint es für Heidegger wie für Rosenzweig möglich, mit der Philosophie auf das auszugreifen, was jenseits der Philosophie liegt. Anders als Heidegger ist Rosenzweig bereit, diese Methode gewordene Bewegung im neuen Denken auf seine eigene Religion zurückzubeziehen. Er reduziert Religion nicht auf das, was von der Philosophie, wie sie sein soll, abgetrennt werden muss, sondern verbindet beides miteinander. Immerhin ist Philosophie methodisch so angelegt, dass sie immer über sich hinausgreift - genau dies aber tut jedenfalls aus Sicht Rosenzweigs Religion doch auch. Als solche aber ist sie, wie eingangs gesagt, mystisch. 


\section{Bibliographie}

Fonti D. (2009), Levinas und Rosenzweig. Das Denken, der Andere und die Zeit, Würzburg, Königshausen \& Neumann.

Heidegger M. (1976), Platons Lehre von der Wahrheit, in Wegmarken, Frankfurt am Mein, Vittorio Klostermann.

- (1986), Sein und Zeit, Tübingen, Max Niemeyer Verlag.

—. (1990), Identität und Differenz, Pfullingen, Neske Verlag.

Prete M. del (2009), Erlösung als Werk. Zur offenbarten Ontologie Franz Rosenzweigs, Freiburg, Karl Alber Verlag.

Rosenzweig Franz (1979), Briefe und Tagebücher, Den Haag, Matirinus Nijhoff.

-. (1988), Der Stern der Erlösung, Frankfurt am Mein, Suhrkamp.

-. (2001), Zweistromland, G. Palmer (hg.), Berlin, Springer Verlag. 